\title{
Effect of topiroxostat in patients with diabetic nephropathy and gout or hyperuricemia: fact or fallacy
}

\author{
Venu Gopal Jonnalagadda ${ }^{1}$ Harish Prabhanna Char $^{2}$
}

Received: 30 January 2018 / Accepted: 5 February 2018 / Published online: 16 February 2018

C) Japanese Society of Nephrology 2018

\section{To the Editor,}

We have read the research article "Uric acid-lowering and renoprotective effects of topiroxostat, a selective xanthine oxidoreductase inhibitor, in patients with diabetic nephropathy and hyperuricemia: a randomized, double-blind, placebo-controlled, parallel-group study (UPWARD study)" by Wada et al. [1], the authors presented comparative study results of topiroxostat and placebo in patients with diabetic nephropathy and hyperuricemia. We would like to request the authors to clarify or elucidate the following points for further understanding.

First, the authors stated that all patients screened $(n=141)$ were with diabetic nephropathy and gout or hyperuricemia. However, Table 1 mentioned as number of patients with type 2 diabetes mellitus (T2DM) in the placebo group $n=21$ out of $n=22$ and in topiroxostat as $n=42$ out of $n=43$, respectively. Hence, patients without T2DM should be excluded from the data analysis set.

Second, at baseline, the values of eGFR in placebo and topiroxostat are $68.3 \pm 21.7$ and $66.3 \pm 17.6 \mathrm{ml} / \mathrm{min} / 1.73 \mathrm{~m}^{2}$, respectively. Based on these values, we can consider them as normal patients, since the eGFR value is more than $60 \mathrm{ml} /$ $\min / 1.73 \mathrm{~m}^{2}$ instead of compromised patients [2].

Third, in Fig. 2 in the placebo group, the number of patients completed as per criteria mentioned is 18 ; however, as per protocol, analysis stated as 19 and number of protocol violation 1 . Considering this, the number of patients as per protocol criteria should have been 17 , but 19 is considered for calculation.

We would be highly obliged for your kind consideration of the above-mentioned clarifications in your article to understand more beneficial effects of a selective xanthine oxidoreductase inhibitor, i.e., topiroxostat in patients with diabetic nephropathy and gout or hyperuricemia.

Funding This paper was not funded.

\section{Compliance with ethical standards}

Conflict of interest All authors declared that they have no potential conflict of interest.

Financial statement The authors have no relevant affiliations or financial involvement with any organization or entity.

\section{References}

1. Wada T, Hosoya T, Honda D, Sakamoto R, Narita K, Sasaki T, et al. Uric acid-lowering and renoprotective effects of topiroxostat, a selective xanthine oxidoreductase inhibitor, in patients with diabetic nephropathy and hyperuricemia: a randomized, doubleblind, placebo-controlled, parallel-group study (UPWARD study). Clin Exp Nephrol. https://doi.org/10.1007/s10157-018-1530-1.

2. What is Glomerular Filtration Rate (GFR) (2018) https://www. kidney.org/atoz/content/gfr. Viewed on Jan 30.
Venu Gopal Jonnalagadda

gopalvenu63@gmail.com

Harish Prabhanna Char

pharish9@gmail.com

1 Department of Pharmacology \& Toxicology, National Institute of Pharmaceutical Education and Research, C/O NETES Institute of Technology \& Science, NH-37, Shantipur, Parli Part, Mirza, Assam 781125, India

2

\footnotetext{
National Institute of Management Studies, 201 Nirman Estate, Link Road, Malad West, Mumbai, Maharashtra 400064, India
} 